\title{
On some geometric characteristics of the orbit foliations of the co-adjoint action of some 5-dimensional solvable Lie groups
}

- Le Anh $\mathrm{Vu}^{1}$

- Nguyen Anh Tuan ${ }^{2}$

- Duong Quang Hoa ${ }^{3}$

\author{
${ }^{1}$ University of Economics and Law, VNU-HCM \\ ${ }^{2}$ University of Physical Education and Sports, Ho Chi Minh city \\ ${ }^{3}$ Hoa Sen University, Ho Chi Minh city \\ (Manuscript Received on August 015t, 2015, Manuscript Revised August 27th 2015 )
}

\section{ABSTRACT:}

In this paper, we discribe some geometric charateristics of the so-called $M D(5,3 C)$-foliations and $M D(5,4)$ -

foliations, i.e., the foliations formed by the generic orbits of co-adjoint action of $M D(5,3 C)$-groups and $M D(5,4)$-groups.

Key words: K-representation, K-orbits, $M D$-groups, $M D$-algebras, foliations.

\section{INTRODUCTION}

It is well-known that Lie algebras are interesting objects with many applications not only in mathematics but also in physics. However, the problem of classifying all Lie algebras is still open, up to date. By the LeviMaltsev Theorem [5] in 1945, it reduces the task of classifying all finite-dimensional Lie algebras to obtaining the classification of solvable Lie algebras.

There are two ways of proceeding in the classification of solvable Lie algebras: by dimension or by structure. It seems to be very difficult to proceed by dimension in the classification of Lie algebras of dimension greater than 6. However, it is possible to proceed by structure, i.e., to classify solvable Lie algebras with a specific given property.

We start with the second way, i.e, the structure approach. More precisely, by Kirillov's Orbit Method [4], we consider Lie algebras whose correponding connected and simply connected Lie groups have co-adjoint orbits (Korbits) which are orbits of dimension zero or maximal dimension. Such Lie algebras and Lie groups are called MD-algebras and MD-groups, respectively, in term of Diep [2]. The problem of classifying general MD-algebras (and corresponding MD-groups) is still open, up to date: they were completely solved just for dimension $n \leq 5$ in 2011 .

There is a noticeable thing as follows: the family of maximal dimension K-orbits of an MDgroup forms a so-called $M D$-foliation. The theory of foliations began in Reeb's work [7] in 1952 and came from some surveys about existence of solution of differential equations [6]. Because of its origin, foliations quickly become a very interesting object in modern geometry.

When foliated manifold carries a Riemannian structure, i.e., there exists a Riemannian metric on it, the considered foliation has much more interesting geometric 
characteristics in which are totally goedesic or Riemannian [8]. Such foliations are the simplest foliations can be on an given Riemannian manifold and have been investigated by many mathematicians. In this paper, we follow that flow to consider some geometric characteristics of foliations formed by K-orbits of indecomposable connected and simply connected MD5-groups whose corresponding MD5-algebras having first derived ideals are 3-dimensional or 4dimensional and commutative.

This paper is organized in 5 sections as follows: we introduce considered problem in Sections 1; recall some results about $\mathrm{MD}(5,3 \mathrm{C})$ algebras and $\mathrm{MD}(5,4)$-algebras in Section 2; Section 3 deals with some results about $\mathrm{MD}(5,3 \mathrm{C})$-foliations and $\mathrm{MD}(5,4)$-foliations; Section 4 is devoted to the discussion of some geometric characteristics of $\mathrm{MD}(5,3 \mathrm{C})$-foliations and $\mathrm{MD}(5,4)$-foliations; in the last section, we give some conclusions.

\section{MD(5,3C)-ALGEBRAS AND MD(5,4)- ALGEBRAS}

Definition 2.1 ([see 4]). Let $G$ be a Lie group and $G$ its Lie algebra. We define an action $A d: G \rightarrow \operatorname{Aut}(\mathrm{G})$ by

$$
\operatorname{Ad}(g):=\left(L_{g} \circ R_{g^{-1}}\right) \text {, }
$$

where $L_{g}$ and $R_{g}$ are left-translation and right-translation by an element $g$ in $G$, respectively. The action $A d$ is called adjoint representation of $G$ in $\mathbf{G}$.

Definition 2.2 ([see 4]). Let $\mathrm{G}^{*}$ be the dual space of $\mathrm{G}$. Then, Ad gives rise an action $K: G \rightarrow \operatorname{Aut}\left(\mathrm{G}^{*}\right)$ which is defined by $\langle K(g) F, X\rangle:=\left\langle F, \operatorname{Ad}\left(g^{-1}\right) X\right\rangle$ for every $F \in \mathrm{G}^{*}$, $X \in \mathrm{G}, g \in G$; where the notation $\langle F, X\rangle$ denotes the value of linear form $F$ at left-invariant vector field $X$. The action $K$ is called co-adjoint representation or $K$-representation of $G$ in $\mathrm{G}^{*}$ and each its orbit is called an $K$-orbit of $\mathrm{S}$ in $\mathrm{G}^{*}$.
Definition 2.3 ([see 2]). An $n$-dimensional $M D$-group or MDn-group is an $\mathrm{n}$-dimensional solvable real Lie group such that its $\mathrm{K}$-orbits in $\mathrm{K}$-representation are orbits of dimension zero or maximal dimension. The Lie algebra of an MDngroup is called MDn-algebra.

Remark 2.4. The family $\mathrm{F}$ of maximal dimension K-orbits of $G$ forms a partition of $V=\cup\{\Omega: \Omega \in \mathrm{F}\}$ in $\mathrm{G}^{*}$. This leads to a foliation as we will see in the next section.

Definition 2.5 ([see 2]). With an MDnalgebra $G$, the $G^{1}:=[G, G$ ] is called the first derived ideal of $\mathbf{G}$. If $\operatorname{dim} \mathrm{G}^{1}=m$, then $\mathrm{G}$ is called an $M D(n, m)$-algebra. Furthermore, if $\mathbf{G}^{1} \cong \square^{m}$, i.e., $\mathbf{G}^{1}$ is abelian, then $\mathbf{G}$ is called an $M D(n, m C)$-algebra.

It is well known that all Lie algebras with dimension $n \leq 3$ are always MD-algebras. For $n=4$, the problem of classifying MD4-algebras was solved by $\mathrm{Vu}$ [10]. Recently, the similar problem for MD5-algebras also has been solved. In this section, we just consider a subclass consists of $\mathrm{MD}(5,3 \mathrm{C})$-algebras and $\mathrm{MD}(5,4)$ algebras. More specifically, we have the following results.

\section{Proposition 2.6 ([10, Theorem 3.1]).}

1)There are 8 families of indecomposable $\mathrm{MD}$ (5,3C)-algebras which are denoted as follows:

$$
\begin{aligned}
& \mathrm{G}_{5,3,1\left(\lambda_{1}, \lambda_{2}\right)} \quad \lambda_{1}, \lambda_{2} \in \square \backslash\{0,1\}, \lambda_{1} \neq \lambda_{2} ; \\
& \mathrm{G}_{5,3,2(\lambda)}, \quad \lambda \in \square \backslash\{0,1\} ; \quad \mathrm{G}_{5,3,3(\lambda)}, \\
& \lambda \in \square \backslash\{1\} ; \mathrm{G}_{5,3,4} ; \mathrm{G}_{5,3,5(\lambda)}, \lambda \in \square \backslash\{1\} ; \\
& \mathrm{G}_{5,3,6(\lambda)}, \lambda \in \square \backslash\{0,1\} ; \mathrm{G}_{5,3,7} ; \mathrm{G}_{5,3,8(\lambda, \varphi)}, \\
& \lambda \in \square \backslash\{0\}, \varphi \in(0, \pi) .
\end{aligned}
$$

2)There are 14 families of indecomposable $\operatorname{MD}(5,4)$-algebras which are denoted as follows:
$\mathrm{G}_{5,4,1\left(\lambda_{1}, \lambda_{2}, \lambda_{3}\right)}$
$\mathrm{G}_{5,4,2\left(\lambda_{1}, \lambda_{2}\right)}$,
$\mathrm{G}_{5,4,3(\lambda)}$,
$\mathbf{G}_{5,4,4(\lambda)}$,
$\mathrm{G}_{5,4,5}$,
$\mathrm{G}_{5,4,6\left(\lambda_{1}, \lambda_{2}\right)}$,
$\mathrm{G}_{5,4,7(\lambda)}$,
$\mathrm{G}_{5,4,8(\lambda)}$,
$\mathrm{G}_{5,4,9(\lambda)}$,
$\mathrm{G}_{5,4,10}$,
$\lambda, \lambda_{1}, \lambda_{2}, \lambda_{3} \in \square \backslash\{0,1\} ;$
$\mathrm{G}_{5,4,11\left(\lambda_{1}, \lambda_{2}, \varphi\right)}$,
$\mathrm{G}_{5,4,12(\lambda, \varphi)}, \mathrm{G}_{5,4,13(\lambda, \varphi)}, \lambda, \lambda_{1}, \lambda_{2} \in \square \backslash\{0\}$, 
$\varphi \in(0 ; \pi) ; \mathrm{G}_{5,4,14(\lambda, \mu, \varphi)}, \lambda, \mu \in \square, \mu>0$, $\varphi \in(0 ; \pi)$.

Remark 2.7. In view of Proposition 2.6, we obtain 8 families of $\mathrm{MD}(5,3 \mathrm{C})$-groups and 14 families of $\mathrm{MD}(5,4)$-groups. All groups of these families are indecomposable, connected and simply connected. For convenience, we will use the same indicates to denote these MD-groups. For example, $G_{5,3,4}$ is the connected and simply connected $\mathrm{MD}(5,3 \mathrm{C})$-group corresponding to $\mathrm{G}_{5,3,4}$.

\section{MD(5,3C)-FOLIATIONS AND MD(5,4)- FOLIATIONS}

Definition 3.1 ([see 1]). A p-dimensional foliation $\mathrm{F}=\left\{L_{\alpha}\right\}$ on an $n$-dimensional smooth manifold $V$ is a family of $p$-dimensional connected submanifolds of $V$ such that:

1) $\mathrm{F}$ forms a partition of $V$.

2)For every $x \in V$, there exist a smooth chart

$$
\varphi=\left(\varphi_{1}, \varphi_{2}\right): U \rightarrow \square^{p} \times \square^{n-p}
$$

defined on an open neighborhood $U$ of $x$ such that if $U \cap L_{\alpha} \neq \varnothing$, then the connected components of $U \cap L_{\alpha}$ are described by the equations $\varphi_{2}=$ const. We call $V$ the foliated manifold, each member of $\mathrm{F}$ a leaf and the number $n-p$ is called the codimension of $\mathbf{F}$.

Let $(V, g)$ be a Riemannian manifold and $\mathrm{F}=\left\{L_{\alpha}\right\}$ be a foliation on $(V, g)$. We denote by $T \mathrm{~F}$ and $N \mathrm{~F}$ the tangent distribution and orthogonal distribution of $F$, respectively.

Definition 3.2 ([see 6, 8]). A submanifold $L \subset V$ is called a totally geodesic if it satisfies one of equivalent conditions as follows:

1) Each geodesic of $V$ that is tangent to $L$ then it lies entirely on $L$.

2) Each geodesic of $L$ is also a geodesic of $V$.

Definition 3.3 ([see 6, 8]). A foliation $F$ on $(V, g)$ is called totally geodesic (and $T \mathrm{~F}$ is called geodesic distribution) if all leaves of $\mathrm{F}$ are totally geodesic submanifolds of $V$. If $N \mathrm{~F}$ is geodesic distribution, then $F$ is called Riemannian.

Remark 3.4. For any foliation $\mathrm{F}$ on $(V, g)$, in the geometric viewpoint, we have

1) $\mathrm{F}$ is totally geodesic if each geodesic of $V$ is either tangent to some leaf of $\mathrm{F}$ or not tangent to any leaf of $\mathrm{F}$.

2) $\mathrm{F}$ is Riemannian if each geodesic of $V$ is either orthogonal to some leaf of $F$ or not orthogonal to any leaf of $\mathrm{F}$.

Definition 3.5 ([see 1]). Two foliations $\left(V_{1}, \mathrm{~F}_{1}\right)$ and $\left(V_{2}, \mathrm{~F}_{2}\right)$ are said to be equivalent or have same foliated topological type if there exist a homeomorphism $h: V_{1} \rightarrow V_{2}$ which sends each leaf of $F_{1}$ onto each leaf of $F_{2}$.

Proposition 3.6 ([see 10, 13, 14]). Let $G$ be one of indecomposable connected and simply connected MD(5,3C)-groups (respectively, $\operatorname{MD}(5,4)$-groups). Let $\mathrm{F}_{G}$ be the family of maximal dimensional K-orbits of $G$, and $V_{G}=\cup\left\{\Omega: \Omega \in \mathrm{F}_{G}\right\}$. Then, $\left(V_{G}, \mathrm{~F}_{G}\right)$ is a measureable foliation (in term of Connes [1]) and it is called $M D(5,3 C)$-foliation (respectively, $M D(5,4)$-foliation) associated to $G$.

Due to Proposition 2.6 and Remark 2.7, there are 8 families of $\mathrm{MD}(5,3 \mathrm{C})$-foliations and 14 families of $\mathrm{MD}(5,4)$-foliations. Note that for all $\mathrm{MD}(5,3 \mathrm{C})$-groups (respectively, $\mathrm{MD}(5,4)$ groups), $V_{G}$ are diffeomorphic to each other. So, instead of $\left(V_{G_{i . . .}}, \mathrm{F}_{G_{i . . .}}\right)$, we will write $\left(V_{i}, \mathrm{~F}_{i, \ldots}\right)$. For example, $\left(V_{3}, \mathrm{~F}_{3,4}\right)$ is $\mathrm{MD}(5,3 \mathrm{C})$-foliation associated to $G_{5,3,4}$.

Proposition 3.7 ([see 10, 14]). With these notations as above, we have: 
1)There exist exactly 2 topological types $F_{1}, F_{2}$ of 8 families of considered $\operatorname{MD}(5,3 C)$ foliations as follows:

$$
\mathrm{F}_{1}=\left\{\left(V_{3}, \mathrm{~F}_{3,1\left(\lambda_{1}, \lambda_{2}\right)}\right),\left(V_{3}, \mathrm{~F}_{3,2(\lambda)}\right), \ldots,\left(V_{3}, \mathrm{~F}_{3,7}\right)\right\}
$$$$
\mathrm{F}_{2}=\left\{\left(V_{3}, \mathrm{~F}_{3,8(\lambda, \varphi)}\right)\right\} .
$$

2) There exist exactly 3 topological types $\mathrm{F}_{3}, \mathrm{~F}_{4}, \mathrm{~F}_{5}$ of 14 families of considered $\mathrm{MD}(5,4)$-foliations as follows:

$$
\begin{aligned}
& \mathrm{F}_{3}=\left\{\left(V_{4}, \mathrm{~F}_{4,1\left(\lambda_{1}, \lambda_{2}\right)}\right),\left(V_{4}, \mathrm{~F}_{4,2(\lambda)}\right), \ldots,\left(V_{4}, \mathrm{~F}_{4,10}\right)\right\} \\
& \mathrm{F}_{4}=\left\{\left(V_{4}, \mathrm{~F}_{4,11\left(\lambda_{1}, \lambda_{2}, \varphi\right)}\right),\left(V_{4}, \mathrm{~F}_{4,12(\lambda, \varphi)}\right),\left(V_{4}, \mathrm{~F}_{4,13(\lambda, \varphi)}\right)\right\} \\
& \mathrm{F}_{5}=\left\{\left(V_{4}, \mathrm{~F}_{4,14(\lambda, \mu, \varphi)}\right)\right\},
\end{aligned}
$$$$
\text { where } V_{3} \equiv \square^{2} \times\left(\square^{3}\right)^{*}, \quad V_{4} \equiv \square \times\left(\square^{4}\right)^{*} \text {. }
$$

\section{SOME GEOMETRIC CHARACTERISTICS OF MD(5,3C)- FOLIATIONS AND MD(5,4)-FOLIATIONS}

Now, we describe some geometric characteristics of considered $\mathrm{MD}(5,3 \mathrm{C})$-foliations and $\mathrm{MD}(5,4)$-foliations.

\subsection{Foliations of the type $F_{1}$}

Choose $F_{3,4}$ represents the type $F_{1}$. From the geometric picture of K-orbits in $[14,15]$, we see that the zero dimensional K-orbits are points in $O x y$, the leaves of $\mathrm{F}_{3,4}$ are 2-dimensional $\mathrm{K}$ orbits as follows:

$$
\Omega_{F}=\left\{\left(\alpha+\left(1-e^{a}\right) \gamma ; y ; e^{a} \gamma ; e^{a} \delta ; e^{a} \sigma\right): y, a \in \square\right\},
$$

where $\gamma^{2}+\delta^{2}+\sigma^{2} \neq 0$.

Recall that $\mathbf{G}_{5,3,4}^{*} \equiv \square^{5}$. Let us identify $O z$ with $\{(0,0)\} \times \square . z \times \square . t \times \square . s$, i.e., each point on $O z$ has coordinate $(0,0, z, t, s)$. So we can see $\mathrm{G}_{5,3,4}^{*}$ as $\square^{3} \equiv O x y z$. Then, all the leaves of $\mathrm{F}_{3,4}$ are half-planes $\{x+z=\gamma+\delta, z>0$ or $z<0\}$ (Figure 1).

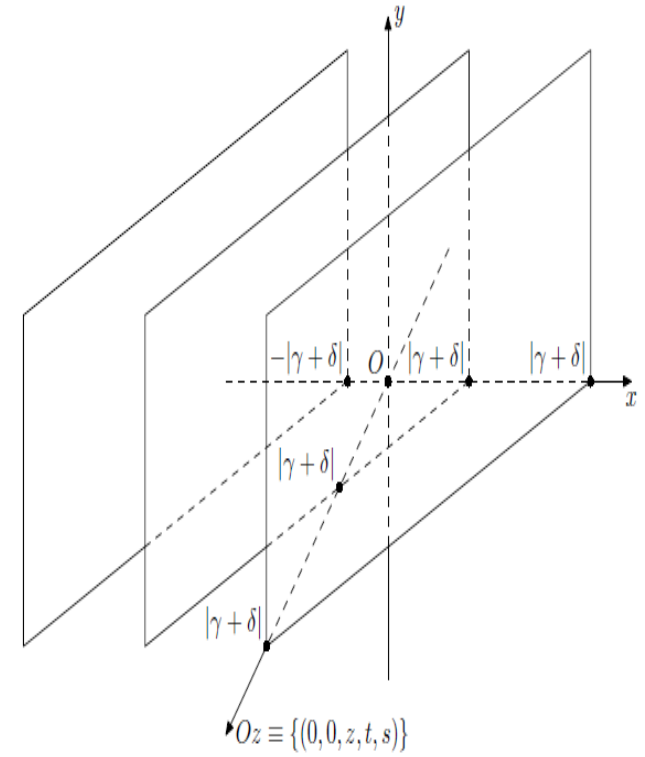

Figure 1. The leaves of $\mathrm{F}_{3,4}$

Because $\quad V_{3} \equiv \square^{2} \times\left(\square^{3}\right)^{*} \quad$ is Euclidean space, its totally geodesic submanifolds are only $k$-planes. Therefore, we have the following proposition.

Proposition 4.1. $\mathrm{F}_{1}$-type $M D(5,3 C)$-foliations are totally geodesic and Riemannian.

\subsection{Foliations of the type $F_{2}$}

Choose $F_{3,8\left(1, \frac{\pi}{2}\right)}$ represents the type $F_{2}$. From the geometric picture of K-orbits in [13, $14]$, we see that the zero dimensional K-orbits are points $F(\alpha, \beta, 0,0,0)$ in $O x y$, the leaves of $\mathrm{F}_{3,8\left(1, \frac{\pi}{2}\right)}$ are 2-dimensional K-orbits $\Omega_{F}=$ $\left\{\left(\alpha+(\sin a) \gamma-(1-\cos a) \delta ; y ;(\gamma+i \delta) e^{-i a} ; e^{a} \sigma\right): y, a \in \square\right\}$,

where $\gamma^{2}+\delta^{2}+\sigma^{2} \neq 0$.

- Let us identify

$$
O y \equiv\{0\} \times \square . y \times \square . z \times \square . t \times\{0\} .
$$

Then, $\mathbf{G}_{5,3,8\left(1, \frac{\pi}{2}\right)}^{*} \equiv \square^{5}$ can be seen as $\square^{3} \equiv$ Oxys. In this case, the leaves of $\mathrm{F}_{3,8\left(1, \frac{\pi}{2}\right)}$ are halfplanes $\{x=\alpha, s>0$ or $s<0\}$ (Figure 2). 


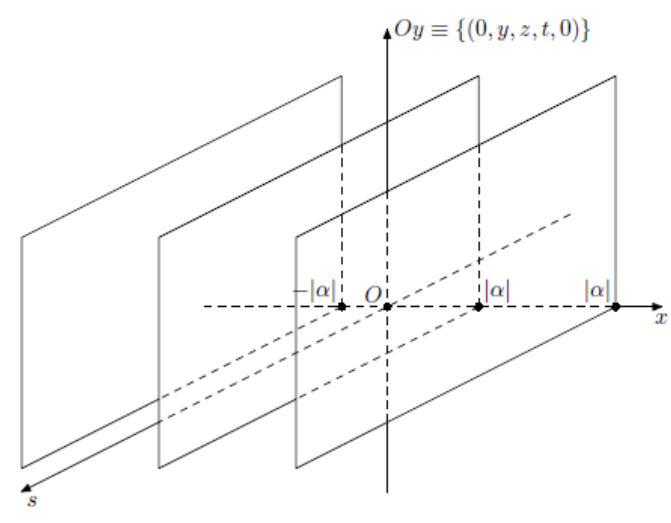

Figure 2. The leaves of $\mathrm{F}_{3,8\left(1, \frac{\pi}{2}\right)}$ in half 3plane $\{z=t=0, \mathrm{~s}>0\}$

- Let us identify

$O t \equiv \square . x \times\{(0,0)\} \times \square . x-\alpha+\delta \times\{0\}$,

Then, $\mathrm{G}_{5,3,8\left(1, \frac{\pi}{2}\right)}^{*}$ can be seen as $\square^{4} \equiv O x y t$. In this case, the leaves of $\mathrm{F}_{3,8\left(1, \frac{\pi}{2}\right)}$ are rotating cylinderes (Figure 3 ).

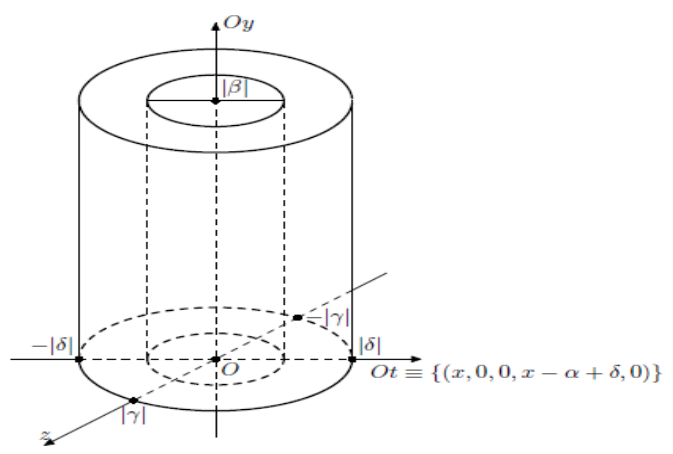

Figure 3. The leaves of , $F_{3,8\left(1, \frac{\pi}{2}\right)}$ in hyperplane

$$
\text { 6.1. } x-t=\alpha-\delta
$$

- Let us identify

$$
O y \equiv\{0\} \times \square . y \times\{(0,0)\} \times \square . s,
$$

and $O t$ as above. Then, $\mathbf{G}_{5,3,8\left(1, \frac{\pi}{2}\right)}^{*}$ can be seen as $\square^{3} \equiv$ Oyzt and the leaves of $\mathrm{F}_{3,8\left(1, \frac{\pi}{2}\right)}$ are cylinderes whose generating curves are parallel to Oy-axis, directrices are helices $\left\{z+i t=(\gamma+i \delta) e^{-i a}, s=e^{a} \sigma\right\}$ in Oyzt.

It is clear that there exist some leaves of $\mathrm{F}_{3,8\left(1, \frac{\pi}{2}\right)}$ which are not totally geodesic submanifolds of $V_{3}$. Therefore, we have the following proposition.

Proposition 4.3. $\quad \mathrm{F}_{2}$-type $M D(5,3 C)$ foliations are not totally geodesic.

\subsection{Foliations of the type $F_{3}$}

Choose $\mathrm{F}_{4,5}$ represents the type $\mathrm{F}_{3}$. From the geometric picture of $\mathrm{K}$-orbits in [10], for $F(\alpha, \beta, \gamma, \delta, \sigma)$ in $\mathrm{V}_{4}$, the leaves of $\mathrm{F}_{4,5}$ are 2dimensional K-orbits as follows:

$$
\Omega_{\mathrm{F}}=\left\{\left(x ; e^{a} \beta ; e^{a} \gamma ; e^{a} \delta ; e^{a} \sigma\right): x, a \in \square\right\},
$$

where $\beta^{2}+\gamma^{2}+\delta^{2}+\sigma^{2} \neq 0$. Let us indentify $O z$ with $\square . z \times \square \cdot \frac{\delta}{\gamma} z \times \square \cdot \frac{\sigma}{\gamma} z$. Then, $\mathrm{G}_{5,4,5}^{*} \equiv \square^{5}$ can be seen as $\square^{3} \equiv O x y z$ and the leaves of $\mathrm{F}_{4,5}$ are half-planes $\gamma y=\beta z$ which rotate around $O x$ (Figure 4).

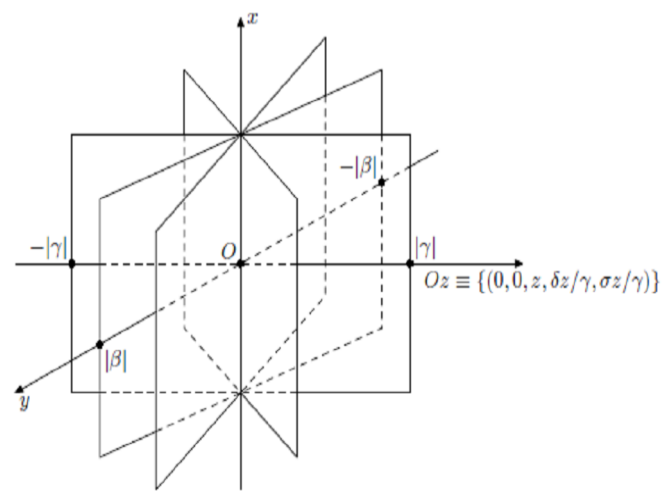

Figure 4. The leaves of $F_{4,5}$

Proposition 4.4. $\quad \mathrm{F}_{3}$-type $M D(5,4)$ foliations are totally geodesic and Riemannian.

\subsection{Foliations of the type $\mathrm{F}_{4}$}

Choose $\mathrm{F}_{4,12\left(1, \frac{\pi}{2}\right)}$ represents the type $\mathrm{F}_{4}$. From geometric picture of K-orbits in [10], for $F(\alpha, \beta, \gamma, \delta, \sigma)$ in $\mathrm{V}_{4}$, the leaves of $\mathrm{F}_{4,12\left(1, \frac{\pi}{2}\right)}$ are 2dimensional K-orbits as follows:

$$
\Omega_{\mathrm{F}}=\left\{\left(x ;(\beta+i \gamma) e^{-i a} ; e^{a} \delta ; e^{a} \sigma\right): x, a \in \square\right\},
$$


where $|\beta+i \gamma|^{2}+\delta^{2}+\sigma^{2} \neq 0$. They are surfaces given by the following cases:

- Let us identify $O x$ with $\square . x \times\{(0,0)\} \times \square . t \times \square . s$. Then, we can see $\mathrm{G}_{5,4,12\left(1, \frac{\pi}{2}\right)}^{*} \equiv \square^{5}$ as $\square^{3} \equiv$ Oxts and the leaves of $\mathrm{F}_{4,12\left(1, \frac{\pi}{2}\right)}$ are half-planes $\sigma t=\delta s$ which rotate around $O x$ (Figure 6).

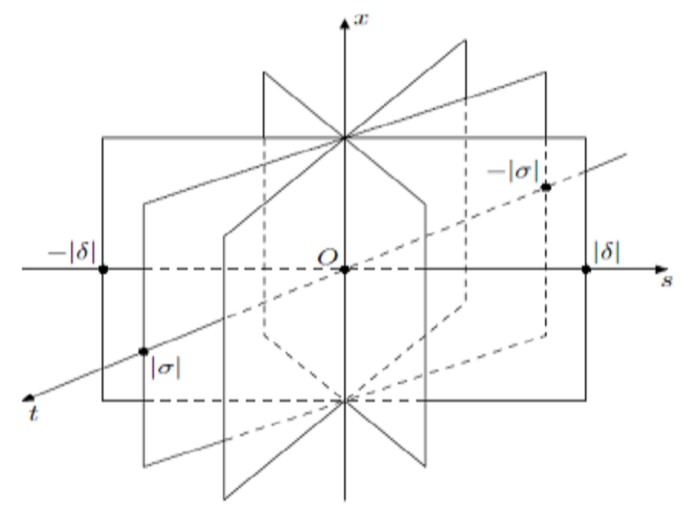

Figure 6. The leaves of $\mathrm{F}_{4,12\left(1, \frac{\pi}{2}\right)}$ in 3-plane

$$
y=z=0
$$

- Let us identify $O x$ with $\square . x \times\{(0,0)\} \times \square . t \times \square . s . \quad$ Then, $\mathrm{G}_{5,4,12\left(1, \frac{\pi}{2}\right)}^{*}$ can be seen as $\square^{3} \equiv O x y z$ and the leaves of $\mathrm{F}_{4,12\left(1, \frac{\pi}{2}\right)}$ are rotating cylinders (Figure 7).

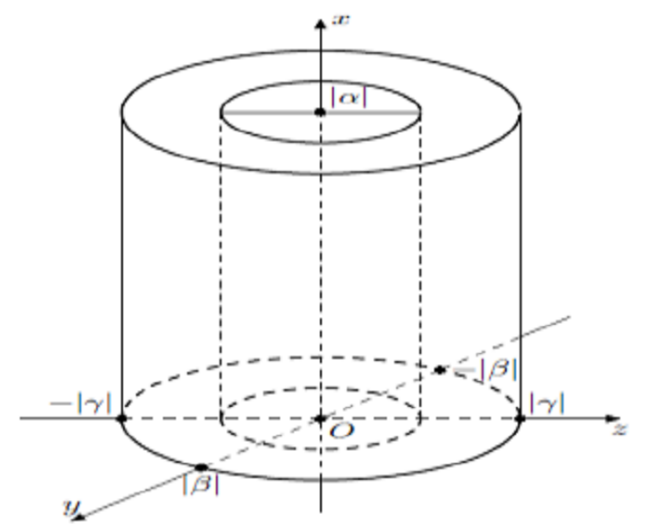

Figure 7. The leaves of $F_{4,12\left(1, \frac{\pi}{2}\right)}$ in 3-plane

$$
t=s=0
$$

- Let us identify $O x$ with $\square . x \times\{(0,0)\} \times \square . t \times \square . \frac{\sigma}{\delta} t$. In this case, the leaves of $\mathrm{F}_{4,12\left(1, \frac{\pi}{2}\right)}$ are rotating cylinders (Figure $8)$.

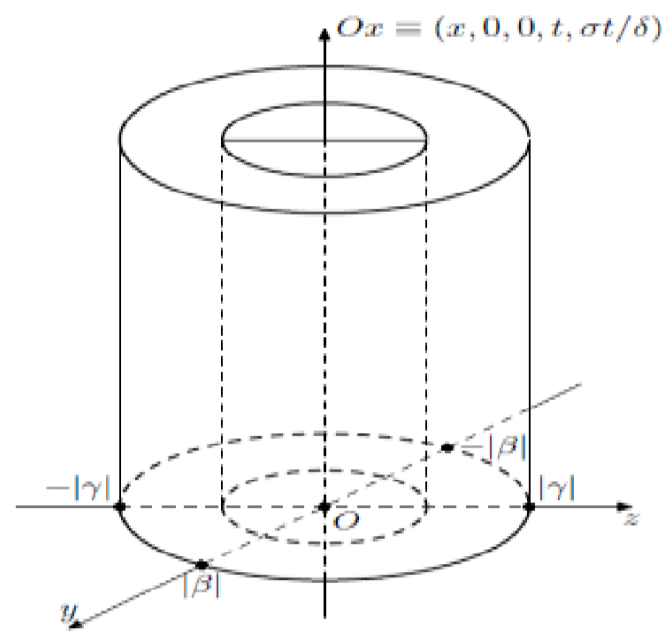

Figure 8. The leaves of $\mathrm{F}_{4,12\left(1, \frac{\pi}{2}\right)}$ in 3-plane

$$
\left\{t=e^{a} \delta, s=e^{a} \sigma\right\}
$$

Proposition 4.6. $\quad \mathrm{F}_{4}$-type $M D(5,4)$ foliations are not totally geodesic.

\subsection{Foliations of the type $F_{5}$}

Choose $F_{4,14\left(0,1, \frac{\pi}{2}\right)}$ represents the type $F_{5}$. From geometric picture of K-orbits in [10], for $F(\alpha, \beta, \gamma, \delta, \sigma)$ in $\mathrm{V}_{4}$, the leaves of $\mathrm{F}_{4,14\left(0,1, \frac{\pi}{2}\right)}$ are 2dimensional K-orbits $\Omega_{F}$ as follows:

$$
\left\{\left(x ;(\beta+i \gamma) e^{-i a} ;(\delta+i \sigma) e^{-i a}\right): x, a \in \square\right\},
$$

where $|\beta+i \gamma|^{2}+|\delta+i \sigma|^{2} \neq 0$. They are surfaces given by each case as follows:

- Let us identify $O z$ with $\{(0,0)\} \times \square . z \times \square . t \times \square . s$. The leaves of $F_{4,14\left(0,1, \frac{\pi}{2}\right)}$ are rotating cylinders (Figure 9). 


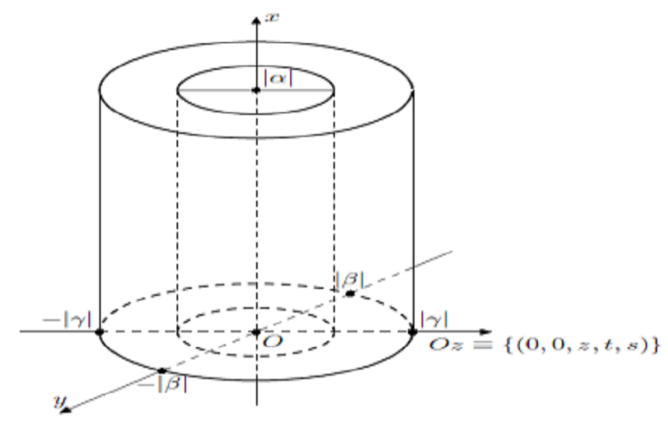

Figure 9. The leaves of $\mathrm{F}_{4,14\left(0,1, \frac{\pi}{2}\right)}$ in 3-plane

$$
t=s=0
$$

- Let us identify $O x$ with $\square . x \times \square . y \times \square . z \times\{(0,0)\}$. The leaves of $\mathrm{F}_{4,14\left(0,1, \frac{\pi}{2}\right)}$ are rotating cylinders (Figure 10).

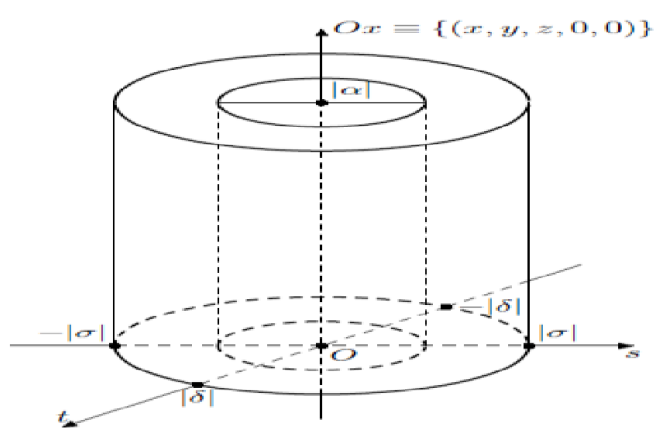

Figure 10. The leaves of $F_{4,14\left(0,1, \frac{\pi}{2}\right)}$ in 3plane $y=z=0$
Finally, that are leaves

$$
\Omega_{F}=\left\{\left(x,(\beta+i \gamma) e^{-i a} ;(\delta+i \sigma) e^{-i a}\right): x, a \in \square\right\} .
$$

Each leaf is a cylinder whose generating curve is parallel to $O x$-axis, directrix is a compact leaf of linear foliation $\mathrm{F}_{1,1}$ [6] on 2dimensional torus $T^{2} \approx S^{1} \times S^{1}$.

Proposition 4.7. $\mathrm{F}_{5}$-type $M D(5,4)$ foliations are not totally geodesic.

\section{CONCLUSION}

In this paper, we described some geometric characteristics of subclass of MD5-foliations: the subclass consists of $\mathrm{MD}(5,3 \mathrm{C})$-foliations and $\operatorname{MD}(5,4)$-foliations. These results gave concrete examples of the simplest foliations on a special Riemannian manifold (Euclidean space). Recently, a special subclass consists of $\operatorname{MD}(n, 1)$ algebras and $\operatorname{MD}(\mathrm{n}, \mathrm{n}-1)$-algebras has been classified for arbitrary $n$. Therefore, in another paper, we will consider a similar problem for the entire class of MD5-foliations; furthermore, for all $\operatorname{MD}(\mathrm{n}, 1)$-foliations and $\operatorname{MD}(\mathrm{n}, \mathrm{n}-1)$-foliations. 


\section{Về một số đặc trưng hình học của các phân lá quỹ đạo tạo bởi tác động đối phụ hợp của một vài nhóm Lie giải được 5- chiều}

- Lê Anh Vũ

- Nguyễn Anh Tuấn ${ }^{2}$

- Dương Quang Hòa ${ }^{3}$

${ }^{1}$ Trường Đại học Kinh tế - Luật, ĐHQG-HCM

${ }^{2}$ Trường Đại học Sư phạm Thể dục Thể thao, TP. Hồ Chí Minh

${ }^{3}$ Trường Đại học Hoa Sen, TP. Hồ Chí Minh

\section{TÓM TÁT:}

Trong bài này, chúng tôi sẽ cho một vài đặc trưng hình học của các $M D(5,3 C)$-phân lá và $M D(5,4)$-phân lá,

tức là các phân lá tạo bởi các quỹ đạo đối phụ hợp ở vị trí tổng quát của các $M D(5,3 C)$-nhóm và $M D(5,4)$-nhóm.

Từ khóa: K-biểu diễn, K-quỹ đạo, MD-nhóm, MD-đại số, phân lá.

\section{REFERENCES}

[1]. A. Connes, A Survey of Foliations and Operator Algebras, Proc. Symp. Pure Math. 38 (I), 512 - 628 (1982).

[2]. D. N. Diep, Method of Noncommutative Geometry for Group $C^{*}$-algebras, Cambridge: Chapman and Hall-CRC Press 1999.

[3]. D. B. Fuks, Foliations, Journal of Soviet Mathematics 18 (2), 255 - 291 (1982).

[4]. A. A. Kirillov, Elements of the Theory of Prepresentations, Springer-Verlag 1976.

[5]. A. I. Maltsev, On solvable Lie algebras, Izvest. Akad. Nauk S.S.R., Ser. Math. 9 (1), $329-356$ (1945).

[6]. P. Molino, Riemannian Foliations, Birkhauser 1988.

[7]. G. Reeb, Sur certains propriétés topologiques de variétés feuilletées, Actualité Sci. Indust. 1183, Hermann 1952.
[8]. P. Tondeur, Foliations on Riemannian Manifolds, Springer-Verlag 1988.

[9]. L. A. Vu, On the foliations formed by the generic K-orbits of the MD4-groups, Acta Mathematica Vietnamica, Vol.15, No2 (1990), 39-55.

[10].L. A. Vu, D.Q. Hoa, The Topology of Foliations Formed by the Generic K-orbits of a Subclass of the Indecomposable MD5groups, Science in China Series A: Mathematics, Vol.52, No2, 351-360 (2009).

[11].L. A. Vu, D. Q. Hoa and N. A. Tuan, KTheory for the Leaf Space of Foliations Formed by the Generic K-orbits of a Class of Solvable Real Lie Groups, Southeast Asian Bulletin of Mathematics 38 (5), 751 770 (2014).

[12].L. A. Vu and K. P. Shum, Classifcation of 5dimensional MD-algebras having commutative derived ideals, Advances in 
Algebra and Combinatorics, Singapore: World Scientific 12 (46), 353 - 371 (2008).

[13].L. A. Vu and D. M. Thanh, The Geometry of K-orbits of a Subclass of MD5-Groups and Foliations Formed by Their Generic Korbits, Contributions in Mathematics and Applications, East-West J. Math. Special Volume, 169 - 184 (2006).
[14].L. A. Vu, N. A. Tuan and D. Q. Hoa, KTheory for the Leaf Spaces of the Orbit Foliations of the co-adjoint action of some 5dimensional solvable Lie groups, East-West J. Math. 16 (2), 141 - 157 (2014).

[15].P. G. Walczak, On foliations with leaves satisfying some geometrical conditions, Polish Scientific Publishers 1983. 